\section{IS THERE A PERFECT SPINAL? A CASE OF SENSORINEURAL HEARING LOSS}

ARSA Silva, TRA Martins*, LAM Ledo, MPS Lucas. Hospital Garcia de Orta, Almada, Portugal

10.1136/rapm-2021-ESRA.117

Background and Aims Spinal anaesthesia (SA) is considered a safe technique even if associated with several complications. Reduced CSF pressure from leakage trough dural puncture site has been associated with post-dural puncture headache $(\mathrm{PDPH})$ and is a proposed mechanism for sensorineural hearing loss (SNHL). Presumably, reduced CSF pressure transmits to inner ear impeding auditory hair cells function. Data reports $0,4 \%$ incidence with some cases of persisting SNHL and correlates with large gauge and cutting point needles utilization. Blood patch, steroid therapy, hyperbaric oxygen and supportive measures are among treatment options with good outcome. We aim to point out a case of isolated SNHL after uneventful SA in a healthy young woman.

Methods 42y healthy woman with uncomplicated pregnancy admitted for elective C-Section at term. SA was successful at first attempt with a $27 \mathrm{G}$ pencil point needle inserted on L3-

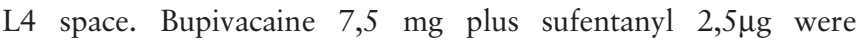
administered after backflow of CSF.

Results No complications were recorded on perioperative period. After 3 days, isolated sudden left SNHL occurred upon standing. Otolaryngologic evaluation showed isolated low frequency left SNHL. Vigorous hydration and restricted supine position resulted in rapid improvement. Audiogram confirmed absence of SNHL at discharged on day 7.

Conclusions SNHL may occur despite an uneventful SA. Improvement after implemented measures reinforces the above-mentioned hypothesis as probable underlaying physiological mechanism. Supportive treatment can be successful managing isolated SNHL. Because there is a risk of permanent damage, a high suspicion index for SNHL should be present even in the absence of PDPH to warrant an early diagnosis.

\section{ANESTHETIC MANAGEMENT FOR CESAREAN SECTION IN A SUPER MORBIDLY OBESE PATIENT}

H Gouveia*, S Reinolds Pereira, J Freitas, R Rodrigues. Serviço de Saúde da Região Autónoma da Madeira - Hospital Central do Funchal, Funchal, Portugal

\subsection{6/rapm-2021-ESRA.118}

Background and Aims Super morbid obesity is defined as a body mass index of $>50 \mathrm{~kg} / \mathrm{m} 2$. Complications from anesthesia and overall morbidity and mortality are higher in this population. Neuraxial techniques are the preferred anesthetic techniques for cesarean delivery in obese parturients but can be technically challenging.

Methods We present the successful anesthetic management of a super morbidly obese obstetric patient with BMI of $68.3 \mathrm{~kg} /$ $\mathrm{m} 2$ underwent cesarean section due to Cephalopelvic disproportion at 38 weeks gestation. A 36-year-old parturient, G2P0 with a history of spontaneous first trimester miscarriage and significant medical history of chronic arterial hypertension, limited mobility, obesity-related shortness of breath and fatigue that had been exacerbated by her pregnancy.

Results In the operating room after several failures to locate the epidural space with an $8 \mathrm{~cm}$ needle, we successfully located it at a depth of $9.5 \mathrm{~cm}$ using a 18 gauge $10 \mathrm{~cm}$

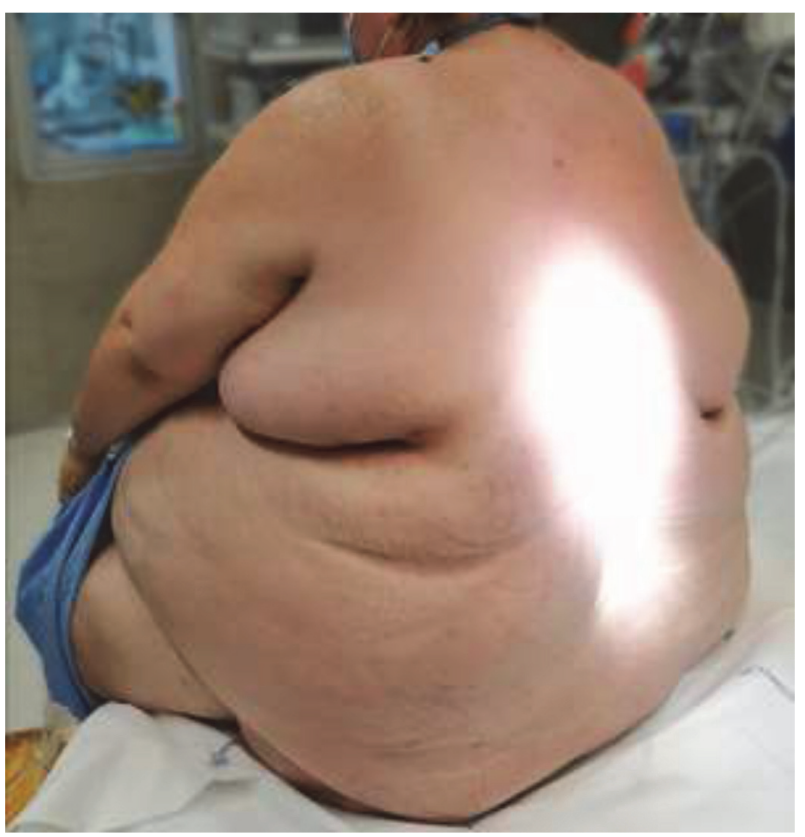

\section{Abstract 118 Figure 1}

Tuohy needle, in the low lombar at an estimated L1-L2 interspace, but a $10 \mathrm{~cm} 24$ gauge quincke neddle for the spinal block wasn't enough. After the placement of the epidural catheter space, we successfully performed the spinal anesthesia at the L3-L4 interspace with a $12 \mathrm{~cm} 22$ gauge and spinal hyperbaric bupivacaine $0.5 \%$ and sufentanyl was administer. The surgery was completed uneventfully. Postoperatively the patient was observed and a multimodal analgesia was satisfactorily provided. The patient was discharged home on postoperative day eight without complications.

Conclusions It is important to report these cases to support the development of new protocols and more adequate conducts for this population.

\section{OUTCOMES OF PREGNANCY AND DELIVERY FOLLOWING A SURGERY OF ADVANCED ENDOMETRIOSIS}

${ }^{1} S$ Krzconavičiūtè ${ }^{*}{ }^{2} V$ Abraitis. ' Lithuanian University of Health Sciences, Kaunas, Lithuania; ${ }^{2}$ Lithuanian University of Health Sciences Kaunas Clinics, Kaunas, Lithuania

\subsection{6/rapm-2021-ESRA. 119}

Background and Aims Even though endometriosis is widely spread among women of reproductive age [1, 2], few researches have been done to identify the influence of endometriosis on pregnancy and delivery outcomes. The aim of this study is to evaluate outcomes of pregnancy and delivery following a surgery of advanced endometriosis.

Methods A retrospective analysis was conducted at LSMU Kaunas Clinics. The sample consisted of 64 women who underwent surgery of endometriosis. The results were compared to the statistical data and other scientific researches. Significant differences were considered when $\mathrm{p}<0.05$.

Results $25 \%$ of pregnancies ended with spontaneous abortion, $15,63 \%$ - with premature labour. Pregnancy complication rate did not statistically significantly differ in IVF pregnant women compared to conceived naturally. More pregnancy complications were identified following a surgery of a III stage of 
endometriosis compared to after surgery of a IV stage of endometriosis $(p<0,05)$. From all analysed deliveries $31,25 \%$ ended with cesarean section. More deliveries via cesarean section were found after IVF compared to natural conception $(p>0,05)$. A higher frequency of cesarean section was found in women who got pregnant after IV stage endometriosis surgery compared to those after III stage surgery $(p>0,05)$.

Conclusions Women after a surgery of III stage endometriosis have an increased probability of pregnancy complications compared to woman after surgery of IV stage endometriosis. Rate of cesarean section is still increased after a surgery of advanced endometriosis. Cesarean sections are performed more frequently on women after surgery of IV stage endometriosis than after surgery of III stage endometriosis.

\section{HOW ELECTRONIC PRESCRIBING BUNDLES IMPROVED PAIN RELIEF PRESCRIBING PRACTICES FOR PATIENTS UNDERGOING ELECTIVE CAESAREAN SECTIONS}

J Patch*, V Pattni, S Bell. Princess Alexandra NHS Trust, Harlow, UK

\subsection{6/rapm-2021-ESRA.120}

Background and Aims The elective caesarean section (CS) rate in England was $15 \%$ in 2019. It is vital to offer adequate pain relief to these patients to improve experience and reduce morbidity. NICE suggests intrathecal/epidural diamorphine for analgesia. If no contraindications, NSAIDs should be offered. RCoA advises that women receiving opioids should have pain scores recorded and anti-emetics and laxatives prescribed.

The prevalence of electronic prescribing in UK hospitals is increasing. These systems can be beneficial in improving patient safety, governance and productivity.

We audited compliance with aforementioned standards and instigated changes to electronic prescribing systems to improve practice.

Methods Data collected prospectively for 26 patients using anaesthetic records, online prescribing charts and pain assessment by questioning patients. Objectives were; 100\% women satisfied with analgesia day 1 post CS; 100\% women receive intrathecal/epidural opioids with regional anaesthetic; Unless contraindicated, 100\% women prescribed NSAIDs; 100\% women prescribed anti-emetics and laxatives PRN.

The cycle was repeated with 15 patients following alteration of prescribing bundles to include recommend medications. Results Initially, $100 \%$ of patients given intrathecal opioid. $69 \%$ received NSAIDs. $19 \%$ prescribed laxatives and $96 \%$ prescribed anti-emetics. $100 \%$ of women expressed satisfaction with pain relief. After modification, opioid use remained $100 \%$. NSAID use rose to $100 \%$. $80 \%$ prescribed laxatives

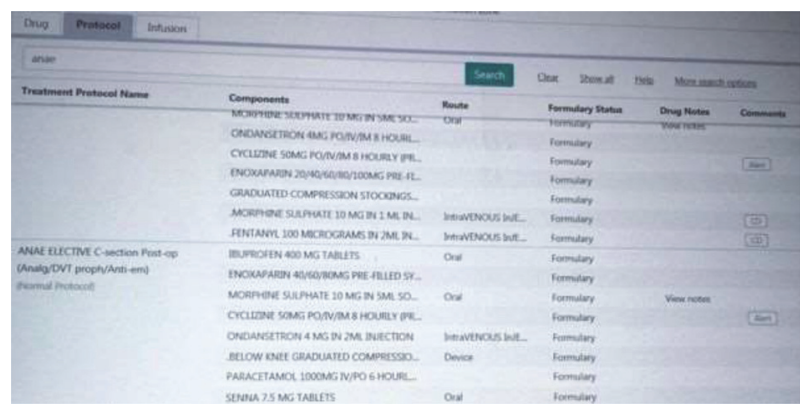

Abstract 120 Figure 1 and $100 \%$ prescribed anti-emetics. Satisfaction remained $100 \%$.

Conclusions Adequate post-operative analgesia is a cornerstone of good anaesthetic practice. Electronic prescribing bundles improved compliance with national standards of recommended analgesia for patients undergoing elective CS.

\section{POSTPARTUM POSTDURAL PUNCTURE HEADACHE: WHEN THINGS CAN GO WRONG..}

K Theodoraki*, I Siafaka. Aretaieion University Hospital, National and Kapodistrian University of Athens, Athens, Greece

\subsection{6/rapm-2021-ESRA.121}

Background and Aims We report a case of cerebral venous sinus thrombosis (CVST) after no obvious dural puncture in a postcesarean section patient

Methods A 32-year old primigravida underwent cesarean section under a combined spinal-epidural. She had a medical history of panic attacks and depression. On the second postpartum day, the parturient developed orthostatic headache which was considered as postdural puncture headache and treated accordingly. Three days postdischarge, the headache returned, having lost its orthostatic component. The parturient however refused to return to hospital for reassessment. Over the next few days, the parturient started behaving strangely and refusing interaction with the newborn. A psychiatrist consulted by the family diagnosed anxiety disorder based on the parturient's previous medical history and suggested anti-anxiety medication.

Results On the seventh postpartum day, the parturient was urgently readmitted to hospital, unable to speak and looking catatonic. Major postpartum depression was diagnosed by the attending psychiatrist and antidepressive medication was initiated. However, on the first readmission night, the parturient developed tonic-clonic seizures and an urgent brain CT-scan revealed multiple left-sided infarcts with haemorrhagic foci. CVST was diagnosed, low-molecular weight heparin was started and the parturient's situation improved dramatically

Conclusions CVST is attributed to cerebral venous dilation and intracranial hypotension in the setting of postpartum hypercoagulability and can have a deleterious outcome if unsuspected and untreated. This case of misdiagnosis emphasizes the need for urgent neurological consultation in the puerperium in case of new or recurrent neurological symptoms. CVST should be considered in the differential diagnosis, especially in case of atypical clinical presentation.

\section{EPIDURAL BLOOD PATCH IN THE $3^{\text {RD }}$ TRIMESTER OF PREGNANCY. WHAT ABOUT LABOUR ANALGESIA?}

P Rebelo, AS Nogueira, C Pinto*. Hospital Pedro Hispano, Matosinhos, Portugal

\subsection{6/rapm-2021-ESRA.122}

Background and Aims Postdural puncture headache (PDPH) is a well-known and common complication of spinal anesthesia which depends upon the characteristics of spinal needle.

Methods We report a blood patch in the 3rd trimester of pregnancy. 